\title{
A Coupled Helicopter Rotor/Fuselage Dynamics Model Using Finite Element Multi-body
}

\author{
Cheng Qi-you, Zhu Yan, Feng Zhi-zhuang, Chen Quan-long \\ Science and Technology on Rotorcraft Aeromechanics Laboratory, China Helicopter Research and Development Institute, Jingdezhen, \\ 333001, China
}

\begin{abstract}
To develop a coupled rotor/flexible fuselage model for vibration reduction studies, the equation of coupled rotor-fuselage is set up based on the theory of multi-body dynamics, and the dynamic analysis model is established with the software MSC.ADMAS and MSC.NASTRAN. The frequencies and vibration acceleration responses of the system are calculated with the model of coupled rotor-fuselage, and the results are compared with those of uncoupled modeling method. Analysis results showed that compared with uncoupled model, the dynamic characteristic obtained by the model of coupled rotor-fuselage are some different. The intrinsic frequency of rotor is increased with the increase of rotational velocities. The results also show that the flying speed has obvious influence on the vibration acceleration responses of the fuselage. The vibration acceleration response in the vertical direction is much higher at the low speed and high speed flight conditions.
\end{abstract}

\section{Introduction}

The structural dynamic analysis of rotorcraft systems involves several interacting elastic components: several blades and rotors, fuselage, and possibly wings. It is convenient to think of such rotorcraft systems as multibody elastic systems, i.e. a collection of mutually interacting elastic body.

As helicopter crew and passenger comfort has gained increased emphasis, vibration requirements have become more stringent [1-3]. Vibration reduction devices are usually used to meet these requirements. Even though there has been enormous progress with vibration suppression technology [4], cost and weight penalty has been excessive in part because of insufficient vibration prediction capability. To minimize the additional cost and weight, accurate vibration prediction is necessary at the early design stage.

A great deal of effort is devoted to improve the mathematical analysis of rotorcraft. Anubhav Datta [5] created a coupled rotor-fuselage analysis model using modal synthesis method. The model was used to analyze the vibration of helicopter. A coupled rotor-fuselage is set up based on finite element method and transfer matrix method [6]. Some author [7-9] developed the analysis model with Finite Element Multibody. However aerodynamic force is ignored in this model. Vellaichamy and Chopra [10] presented a coupled rotor-fuselage analysis using elastic blade and flexible fuselage modeling (stick model). Chiu [11] and Yeo [12] developed coupled rotor/flexible fuselage model for vibration reduction studies using 3-D fuselage. Euler and
Navier-Stokes CFD codes have begun to be applied to the problem [13,14]. A recent rotor-body interactional aerodynamics study by Wachspress, Quackenbush and Boschitsch [15] shows the current state of the art. Interactional effects were studied using a full span constant vorticity contour free wake and a panel method to model the fuselage bodies and lifting surfaces. However, these analyses incorporated highly idealized aerodynamics such as uniform or linear inflow and quasisteady aerodynamics so that vibration was substantially under predicted.

The present study is aimed at developing a coupled rotor/flexible fuselage model for vibration reduction studies. The equation of coupled rotor-fuselage is set up based on the theory of multi-body dynamics, and the dynamic analysis model is established with the software MSC.ADMAS and MSC.NASTRAN. The frequencies and vibration acceleration responses of the system are calculated with the model of coupled rotor-fuselage, and the results are compared with those of uncoupled modeling method. The influence of whirling speed on the intrinsic frequency and the influence of speed on vibration are also analyzed.

\section{Theory}

\subsection{Coordinate systems}

Several coordinate systems are used to describe blade sectional velocity or position vector. Fig.1 illustrates the 
ground-fixed, hub and fuselage coordinate systems. The definition of these coordinate systems is shown below:

1. The ground-fixed inertial coordinate system $\{\mathrm{Og}$, $\mathrm{Xg}, \mathrm{Yg}, \mathrm{Zg}\}$, which is fixed to the ground and does not move with the helicopter. Its unit vector is $\{\mathrm{ig}, \mathrm{jg}, \mathrm{kg}\}$. And the gravity has the same direction of $-\mathrm{kg}$.

2. The fuselage-fixed coordinate system $\{\mathrm{Of}, \mathrm{Xf}, \mathrm{Yf}$, $\mathrm{Zf}\}$,with unit vector $\{$ if, jf, kf\} fixed at the helicopter center of gravity. And six parameters ( Xf, Yf, Zf, $\phi$ Xf, $\phi Y f, \phi Z f)$ are definite to describe the position of fuselage relate to the ground-fixed inertial coordinate system.

3. The hub-fixed coordinate system $\{\mathrm{OH}, \mathrm{XH}, \mathrm{YH}$, $\mathrm{ZH}\}$, with unit vector $\{\mathrm{iH}, \mathrm{jH}, \mathrm{kH}\}$ fixed at the hub. When there are several rotor system, the hub-fixed coordinate system can be definite as $\{\mathrm{OH}$ i, XH i, YH i, ZH i $\} \quad(i=1,2, \cdots, I R)$.

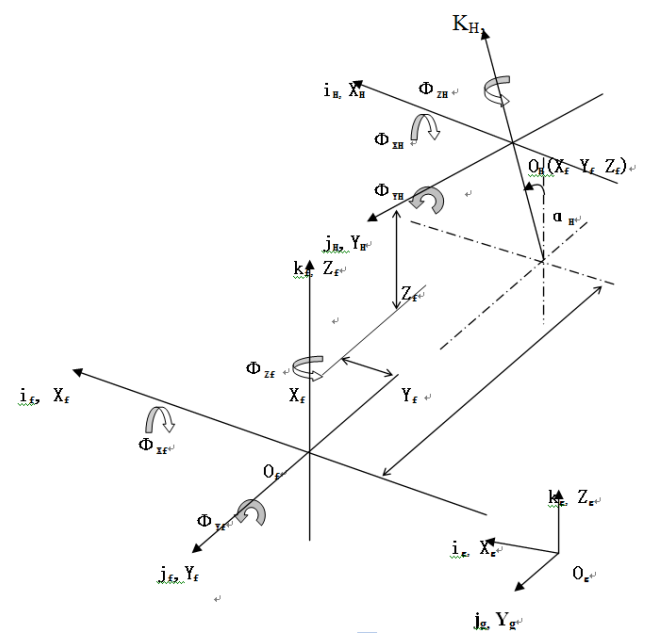

Figure 1. Coordinate systems

\subsection{Mathematical Model}

According to the definition of coordinate system, $r_{n o}$ and $\mathrm{R}_{\mathrm{np}}$ are definite to describe the position of the origin point and random point on the blade to the ground-fixed inertial coordinate system. The Rnp can be expressed as:

$$
R_{n p}=r_{n o}+A_{n o}\left(r_{n p}+u_{n p}\right)
$$

Where, $A_{n o}$ is the position matrix which the fuselagefixed coordinate system relative to the ground-fixed inertial coordinate system. $r_{n p}$ is position vectors without deformation of point $p$. The $u_{n p}$ is the deformation of point $\mathrm{p}$.

The position matrix of rotor-fixed coordinate system can be written as follows:

$$
A_{n o}=A_{F} A_{H} A_{S} A_{P} A_{L} A_{T}
$$

Where $A_{f}, A_{H}, A_{S}, A_{P}, A_{L}, A_{T}$ is position matrix of fuselage, hub, blade, flapping motion of blade, lagging motion of blade, and twist of blade, respectively.

The blade can be view as a several discrete elements, and the deformation of blade can be descript by the location of these elements. At the direction of small elastic deformation, the displacement of these elements can be transformed to the modal domain, using a truncated number of free vibration mode shapes. The deformation of point $\mathrm{P}$ can be written as follows:

$$
u_{n p}=\phi_{i} h
$$

Where $\phi_{i}$ and $\mathrm{h}$ is mode shapes component and amplitude of node I, respectively.

We now substitute Eq.(3) into the Eqs.(1). We obtain the following relations:

$$
R_{n p}=r_{n o}+A_{n o}\left(r_{n p}+\phi_{i} h\right)
$$

It is definite that:

$$
\begin{gathered}
r_{n o}=[x, y, z]^{T} \\
\Psi=[\psi, \theta, \phi]^{T} \\
h=\left[h_{1}, h_{2}, \cdots, h_{m}\right]^{T}
\end{gathered}
$$

Where, $x, y, z$ is coordinate of blade origin in the ground-fixed inertial coordinate system. $\psi, \theta, \phi$ is Euler angle of the blade-fixed inertial coordinate system relative to the ground-fixed inertial coordinate system. $\mathrm{h}_{\mathrm{m}}$ is vibration component of m-order amplitude.

The object of blade motion can be written as

$$
\xi=\left[r_{n o}^{T}, \Psi^{T}, h^{T}\right]^{T}
$$

The velocity of node i can be obtained

$$
v_{i}=\left[I,-A_{n o}\left(\widetilde{r}_{n o}+\widetilde{\phi}_{i} h\right) B, A_{n o} \phi_{i}\right] \dot{\xi}
$$

Where $\tilde{r}_{n o}, \widetilde{\phi}_{i}$ are position vector, and $\mathrm{B}$ is transform matrix.

From Eq.(7), the kinetic energy and potential energy can be obtained

$$
\begin{gathered}
T=\frac{1}{2} \sum_{i=1}^{I} m_{i} v_{i}^{T} v_{i}=\frac{1}{2} \dot{\xi}^{T} M(\xi) \dot{\xi} \\
V=\frac{1}{2} \dot{\xi}^{T} K(\xi) \dot{\xi}
\end{gathered}
$$

Where, The $\mathrm{M}$ is mass matrix. $\mathrm{K}$ is stiffness matrices.

Taking Eq.(8) and Eq.(9) into the Lagrangian equation, We obtain the following relations

$$
M \ddot{\xi}+\dot{M} \dot{\xi}-\frac{1}{2}\left[\frac{\partial M}{\partial \xi} \dot{\xi}\right]^{T} \dot{\xi}+K \xi+f_{g}+D \dot{\xi}+\left[\frac{\partial \Omega}{\partial \xi}\right] \lambda=Q
$$

Where, $\mathrm{D}$ is damping matrix, $f_{g}$ is extended gravity, $\lambda$ is Lagrange multiplier, $\Omega$ and $Q$ are loads.

\subsection{Model}

The baseline rotor analysis is taken from MSC.ADAMS. The blade is assumed to be an elastic beam undergoing flap bending, lag bending, elastic twist, and axial deformation. The elastic rotor coupled equations include six hub degrees of motion. The rotor vibratory loads are transmitted to the fuselage through the hub and the effects of fuselage motion are included in the determination of blade loads.

The elastic fuselage is represented by a complete three dimensional finite element model, and a collection of elements (i.e. element library) is used to generate the structural dynamic model of the fuselage. The elements available are: beam, truss, non-structural mass, and a plate element. The concentrated non-structural masses of the helicopter such as transmission engine, fuel tanks, 
gearing, and payload are modeled using consistently derived finite element model for inertia properties. The non-structural mass element is capable of three translational displacements as well as three rotational degrees of freedom at each node. Due to the large number of degrees of freedom present in the finite element model of the fuselage, the equations are transformed to the modal domain, using a truncated number of free vibration mode shapes. The airframe modal data (eigenvalue, eigenvector, and generalized mass) are generated using NASTRAN and are used as an input to the coupled rotor/fuselage vibration analysis program. The couplings between rotor and fuselage are included in a consistent manner into the MSC.ADAMS.

\section{Results and discussion}

A civil helicopter is chosen for this study. The frequencies and vibration acceleration responses of the system are calculated with the model of coupled rotorfuselage, and the results are compared with those of uncoupled modeling method.

Displayed in Table 1 is the frequency of rotor system with different rotational velocities. It is observed that the rotational velocity has obviously influence on the frequency. The frequency is increased with the rotational velocity. This is because the modulus of blades increases as rotational velocity increases. The calculate results are in accordance with the test results. However, the calculate results are slightly above the test results. This is because the restraint stiffness of calculate is slightly more than the test.

It is also observed that the results obtained with coupling method are slightly less than the one obtained with isolated rotor. This is because the restraint stiffness of rotor system with coupling method is slightly more than with isolated rotor.

Table 1. Rotor system frequency vs rotational velocities

\begin{tabular}{|c|c|c|c|c|}
\hline \multicolumn{2}{|c|}{ rotational velocities (r/min) } & $\mathbf{2 9 0}$ & $\mathbf{3 2 8}$ & $\mathbf{3 8 6}$ \\
\hline \multirow{2}{*}{$\begin{array}{c}\text { Isolated } \\
\text { Rotor }\end{array}$} & calculate/Hz & 14.11 & 15.53 & 18.53 \\
\cline { 2 - 5 } & experiment/Hz & 13.63 & 15.24 & 18 \\
\hline $\begin{array}{c}\text { Coupled } \\
\text { method }\end{array}$ & calculate $/ \mathbf{H z}$ & 13.27 & 14.58 & 17.3 \\
\hline \multicolumn{2}{|c|}{ comparison of two computation } & $5.82 \%$ & $6.12 \%$ & $6.64 \%$ \\
\hline
\end{tabular}

The points selected for vibration suppressions are located on rudder pedal (P1 and P2), flight-deck floor (P3 and $\mathrm{P} 4)$, cockpit floor (P5), and main jackstay (P6 and P7).

The vibration acceleration responses without vibration-absorbing system of direction $\mathrm{X}, \mathrm{Y}$, and $\mathrm{Z}$ in the points selected for vibration suppression are shown in Figure 2.

The results show that the vibrations in the direction $X$ and $\mathrm{Y}$ are relatively low. However the vibration acceleration response in the vertical direction is much higher than the other directions. It is means that the vibration suppression in the vertical direction is the most important. So the next discussion on the actuator optimization selection is focus on the vertical direction.

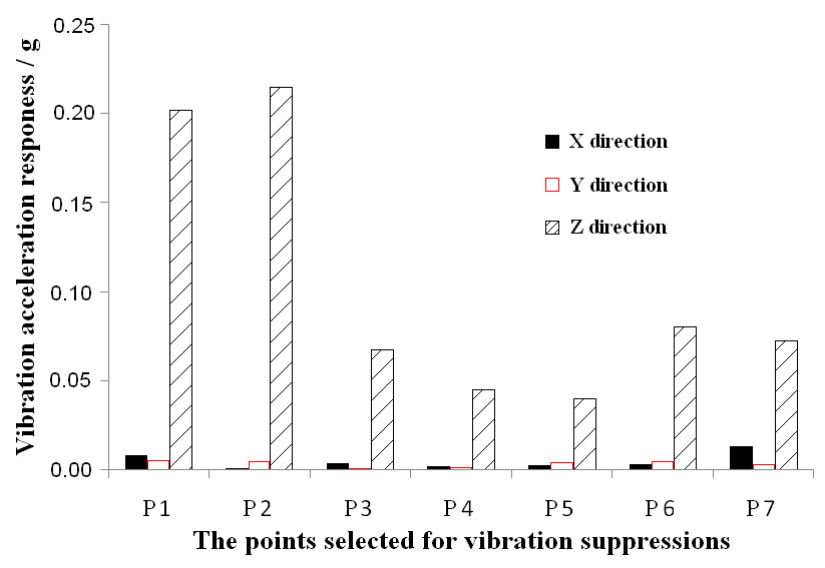

Figure 2. Vibration acceleration responses

The vibration acceleration responses of vertical direction in the key points of fuselage obtained with coupling method and uncoupled method are shown in Figure 3.

It is also observed that the results obtained with coupled method are slightly higher than the one obtained with uncoupled method. It is because the influence of rotor system on the infrequency of fuselage is ignored in the analysis of fuselage with the finite element method. However the frequencies of fuselage obtained with these two methods are different. If the frequency of fuselage obtained with coupling method is tend to the frequency of external load, the vibration acceleration responses will increase. Conversely, the vibration acceleration responses will decrease.

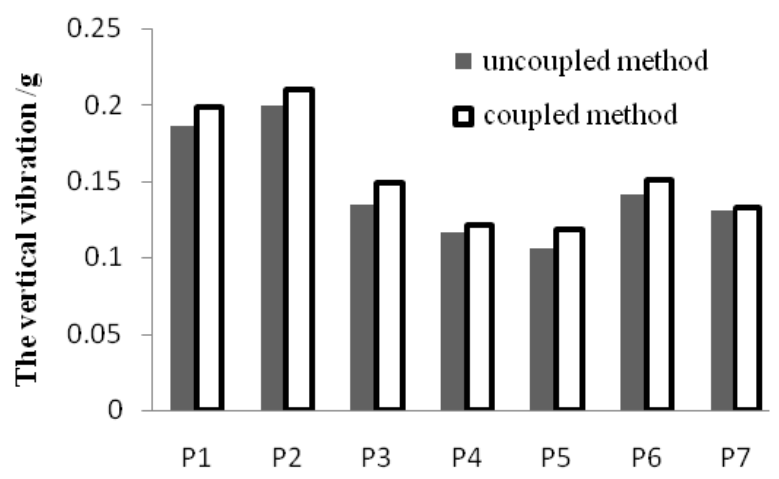

The points selected for vibration suppressions

Figure 3. Vibration acceleration responses of computation

The influence of velocity on the vertical vibration in the key points of fuselage are shown in Figure 4. Three different speeds $(75,120$, and $221 \mathrm{~km} / \mathrm{h})$ which respectively represent low speed, moderate speed, and high speed flight conditions.

The results show that there are some differences on the vibration at different flight speed. The vibration acceleration response in the vertical direction is much higher at the low speed and high speed flight conditions.

At the low speed conditions, the unequal spaced distribution of induced velocity is the main factor of serious vibration. And the vortex wake from the main rotor impinges directly on the tail boom that gives rise to fuselage vibration at the blade passage frequency. 
Under the high speed flight condition, the vertical vibration is much higher. The airflow separation and contraction is the cause of vibration at the high speed flight condition.

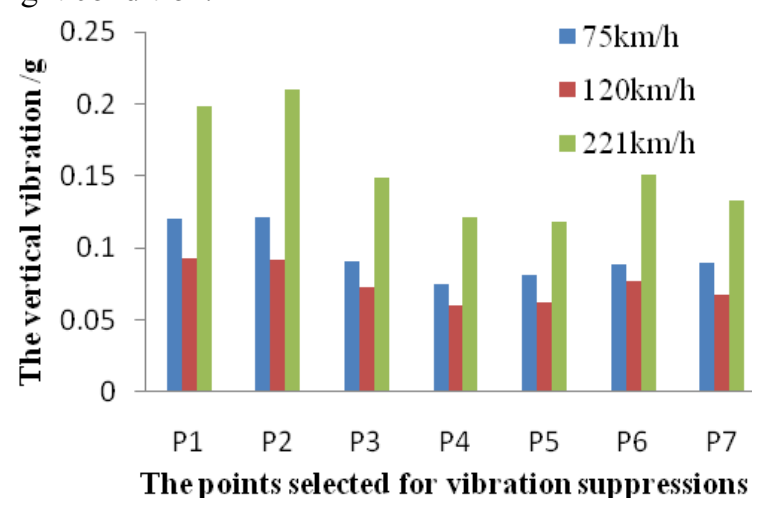

Figure 4. Influence of velocity on the vibration

\section{Conclusions}

The equation of coupled rotor-fuselage is set up based on the theory of multi-body dynamics, and the dynamic analysis model is established with the software MSC.ADMAS and MSC.NASTRAN. The frequencies and vibration acceleration responses of the system are calculated with the model of coupled rotor-fuselage, and the results are compared with those of uncoupled modeling method. Findings are listed below:

1) The calculate results are in accordance with the test results. However, the calculate results are slightly above the test results. This is because the restraint stiffness of calculate is slightly more than the test.

2) With the increase of rotational velocities, the modulus of blades is increased which make the intrinsic frequency of rotor increased.

3) The dynamic characteristics obtained by the model of coupled rotor-fuselage are some different from the results obtain by uncoupled model. If the frequency of fuselage obtained with coupling method is tend to the frequency of external load, the vibration acceleration responses will increase. Conversely, the vibration acceleration responses will decrease.

4) The flying speed has obvious influence on the vibration acceleration responses of the fuselage. The vibration acceleration response in the vertical direction is much higher at the low speed and high speed flight conditions. The unequal spaced distribution of induced velocity is the main factor of serious vibration at the low speed flight condition. However, the airflow separation and contraction is the cause of vibration at the high speed flight condition.

\section{References}

1. Crews, S. T., "Rotorcraft Vibration Criteria A New Perspective," Proceedings of the 43rd Annual Forum of the American Helicopter Society, St. Louis, MO, May 1987.
2. Chopra, I., "Status of Application of Smart Structures Technology to Rotorcraft Systems," Journal of the American Helicopter Society, Vol. 45, No. 4, October 2000, pp 228-252.

3. Roget, B., Chopra, I., "Wind Tunnel Testing of an Individual Blade Controller for a Dissimilar Rotor," Presented at the American Helicopter Society $60^{\text {th }}$ Annual Forum, Baltimore, MD, June 7-10, 2004.

4. Reichert, G., "Helicopter Vibration Control-A Survey," Vertica, Vol. 5, No. 1, 1981.

5. Anubhav Datta, "FUNDAMENTAL UNDERSTANDING, PREDICTION AND VALIDATION OF ROTOR VIBRATORY LOADS IN STEADY LEVEL FLIGHT," Ph.D. dissertation, Dept. Elect. Eng., Harvard Univ., Cambridge, MA, 2004.

6. Hohenmeser K H, Yin S K., "The role of rotor impedance in the vibration analysis of rotorcraft," Vertica, Vol. 3, No. 2, pp. 187-204, 1979.

7. Bianchi F. Helicopter Tail Rotor ANALYSIS EXPERIENCE IN AGUSTA WITH ADAMS, Agusta, 1994.

8. Olivier A. Bauchau.,Carlo L. Bottasso., "T Modeling Rotorcraft Dynamics with Finite Element Multibody," Mathematical and Computer Modeling, pp. 1113-1137, 2001.

9. Altorio Bertogalli, Francesco Bisi., Marco Lovera. MODELLING HELICOPTER ROTOR DYNAMICS WITH ADAMS: 1995.

10. Vellaichamy, S., Chopra, I., "Effect of Modeling Techniques in the Coupled Rotor-Body Vibration Analysis," Proceedings of the 34th Structures, Structural Dynamics and Materials Conference and Adaptive Structures Forum, LaJolla, April 1993.

11. Chiu, T., Friedmann, P. P., "A Coupled Helicopter Rotor/Fuselage Aeroelastic Response Model for ACSR," Proceedings of the 36th Structures, Structural Dynamics and Materials Conference and Adaptive Structures Forum, New Orleans, April 1995.

12. Yeo, H., Chopra, I., "Coupled Rotor/Fuselage Vibration Analysis Using Detailed 3-D Airframe Models," Mathematical and Computer Modelling 33 (2001) pp. 1035-1054.

13. Zori, L. A. J., Mathur, S. R., Rajagopalan, R. G., "Three Dimensional Calculations of Rotor-Airframe Interaction in Forward Flight," american Helicopter Society 52nd Annual Forum Proceedings, Washington D.C., June 1992.

14. Bettschart, N., "Rotor Fuselage Interaction : Euler and Navier-Stokes Computations with an Actuator Disk," American Helicopter Society 55th Annual Forum Proceedings, Montreal, Canada, May 1999.

15. Wachspress, D. A., Quackenbush, T. R., Boschitsch, A. H., "Rotorcraft Interactional Aerodynamics with Fast Vortex/Fast Panel Methods," Journal of the American Helicopter Society, Vol. 48, No. 4, October 2003. 